\section{International Scientific Journal Theoretical \& Applied Science}

\author{
p-ISSN: 2308-4944 (print) e-ISSN: 2409-0085 (online) \\ Year: 2017 Issue: 09 Volume: 53
}

Published: $21.09 .2017 \quad \underline{\text { http://T-Science.org }}$

SECTION 2. Applied mathematics. Mathematical modeling.
Mohiniso Hidirova

$\mathrm{PhD}$, Senior Scientist of dept. "Regulatorika"

Science and innovation center for information and communication technologies at the Tashkent University of Information Technologies named after Muhammad Al-Khwarizmi regulatorika@yahoo.com

Alisher Shakarov Scientist of dept. "Regulatorika" Science and innovation center for information and communication technologies at the Tashkent University of Information Technologies named after Muhammad Al-Khwarizmi regulatorika@yahoo.com

\title{
MATHEMATICAL MODELING REGULATORY MECHANISMS OF IMMUNE REACTIONS AT SKIN ANOMALIES
}

Abstract: This paper considers the method for modeling regulatorika of the skin's immune system. under various anomalies. The developed equations for a quantitative study of the mechanisms of origin, development and consequences of concrete skin diseases make it possible to analyze possible ways for its early diagnosis, forecasting and selection of treatment strategies by means of mathematical and computer modeling.

Key words: modeling, regulatorika, functional-differential equations with delay, skin.

Language: Russian

Citation: Hidirova M, Shakarov A (2017) MATHEMATICAL MODELING REGULATORY MECHANISMS OF IMMUNE REACTIONS AT SKIN ANOMALIES. ISJ Theoretical \& Applied Science, 09 (53): 119-124.
Soi: http://s-o-i.org/1.1/TAS-09-53-18
Doi: crossef https://dx.doi.org/10.15863/TAS.2017.09.53.18

\section{УДК 576.35:517.948}

\section{МАТЕМАТИЧЕСКОЕ МОДЕЛИРОВАНИЕ РЕГУЛЯТОРИКИ ИММУННЫХ РЕАКЦИЙ ПРИ КОЖНЫХ АНОМАЛИЯХ}

Аннотация: В статье рассматривается метод моделирования регуляторики иммунной системь кожи при различных аномалиях. Разработанные уравнения для количественного исследования механизмов возникновения, развития и последствий конкретных кожных заболеваний позволяют проводить анализ возможных путей их ранней диагностики, прогнозирования и выбора стратегий лечения средствами математического и компьютерного моделирования.

Ключевые слова: моделирование, регуляторика, функиионально-дифференцииальнье уравнения с запаздыванием, кожа.

\section{Introduction}

Актуальность проблемы кожных заболеваний (особенно кожных заболеваний иммунного характера и рака кожи) на сегодняшний день не только не уменьшается, а наоборот только возрастает и обусловлена высокой социально-экономической значимостью этого заболевания, поскольку оно широко распространяется среди лиц трудоспособного, репродуктивного возраста, а это приводит к значительным расходам государства на лечение пациентов. На данный момент этиология и патогенез многих кожных заболеваний остаются неизвестными и поэтому весьма актуальными становятся исследования этих процессов путем математического моделирования. Применение современных медицинских информационных технологий обеспечивает эффективность ранней диагностики и профилактики кожных заболеваний. Методы качественных и количественных исследований на основе математического и компьютерного моделирования приобретают все большее значение, становясь одним из основных методов анализа живых систем, поскольку новые технологии и экспериментальные методики привели к огромному росту количества информации о живых объектах. На основании такого большого объема данных становится затруднительным, а порой и невозможным делать 
выводы о работе сложных систем, а тем более делать предсказания и выдвигать конкретные предположения. Ограниченным аналитическим способностям человека здесь на помощь пришли математические и компьютерные методы. Математическое моделирование является наиболее мощным и универсальным теоретическим методом в биологии и медицине. Любая модель, претендующая, в рамках рассматриваемых задач, на некоторую адекватность своему объекту и обладающая предсказательной способностью, должна быть разработана исходя из твердо установленных данных о рассматриваемой системе. Чем сложнее модель, тем большее количество экспериментальных данных необходимо использовать. Как правило, при определении значений параметров используются алгоритмы численной подгонки под экспериментально наблюдаемые биологические закономерности. Данный подход широко распространен, однако он не позволяет решать поставленные задачи в полном объеме. Кроме того, при данном подходе многие теоретические результаты относительно моделируемого явления остаются за пределами количественных исследований

по рассматриваемому методу.

\section{Materials and Methods}

В настоящее время применяются различные подходы математического описания функционирования иммунной системы кожи посредством обыкновенных дифференциальных уравнений, уравнений в частных производных, вероятностных уравнений, функциональнодифференциальных, функциональных и дискретных уравнений [1-8]. Проводятся компьютерные исследования, путем вычислительных экспериментов, на основе имитационных моделей иммунной системы эпидермиса кожи. Но при этом эффективных, общеизвестных методов построения уравнений динамики иммунной системы кожи с учетом регуляторной роли цитокинов, методов их качественного и количественного исследования для осуществления достаточно адекватного описания процессов в организме человека еще не существуют. В последнее время указанные недостатки существующих методов моделирования на основе текущих экспериментальных данных привели к развитию моделирования биологических процессов с точки зрения теории и методов динамических систем, основное внимание при этом уделяется функционированию регуляторных механизмов (регуляторике) рассматриваемого явления $[9,10]$. Анализ существуюих подходов математического моделирования регуляторных механизмов живых систем показал, что общей чертой рассматриваемых модельных исследований является количественный анализ поведения комплекса элементов, функционирующих в некоторой среде и способных реагировать на определенные внешние воздействия. Это приводит [11] к формулировке понятий or (оператороврегуляторов) - элементов регуляторной системы, способных к восприятию и синтезу сигналов определенной природы, и asta (active system with time average) - сигнальной среды регуляторной системы, в которой взаимосвязанная деятельность элементов осуществляется, на основе обратных связей, с некоторым средним временем $h$ (временем, прошедшим с момента образования сигналов до момента воздействия их (или их продуктов) на активность элементов). $\boldsymbol{O r}$ вместе с asta составляют регуляторную систему orasta. Геометрия подобных регуляторных систем является динамической, в которой понятие неподвижной точки теряет смысл. Единицей времени orasta является $h$. Функционирование регуляторных механизмов подобных систем, для краткости, обозначены термином «регуляторика» [11]. По определению Б.Н. Хидирова - Регуляторикой в широком смысле этого слова называется наука, посвящённая решению любых задач, связанных с изучением регуляторных механизмов материи. Теоретическая регуляторика представляет собой часть регуляторики, в которой изучаются общие законы и регуляторные механизмы. В природе наблюдаются случаи раздельного существования or и asta. Примером or без asta являются вирусы, а asta без or - зрелые безъядерные эритроциты. Таким образом, данный подход дает возможность использовать только твердо установленные биологические факты и теоретические положения с учетом широкого диапазона значений параметров и начальных состояний в фазовом и параметрическом пространствах. Здесь «математически обслуживается» не один отдельно взятый факт или набор экспериментальных данных, а большие классы экспериментальных и теоретических результатов, объединенных в единое целое и сформулированных в виде закономерностей проявления регуляторных механизмов данного процесса или явления с учетом наиболее существенных, основных факторов в форме минимальной компактной «биологической модели». Рассмотрим биологическую модель иммунной системы кожи.

Кожа, являясь внешним покровом тела, выполняет ряд функций, таких как терморегуляция, выделительная, дыхательная, обменная и защитная. Эпидермис представляет собой наружный слой кожи и состоит из клеток 
рогового, блестящего, зернистого, шиповатого и базального или росткового слоев (рисунок). Эпидермис является биологически наиболее активным слоем кожи и тесно соединен с дермой, вдавливаясь в нее отростками в виде сосочков. Меланоциты $\left(\mathrm{S}_{1}\right)$ происходят из эмбрионального нервного гребешка, откуда мигрируют к органам и тканям. Различают несколько стадий дифференцировки меланоцитов, в эпидермисе происходит дендритическая стадия, когда меланоцит формирует свои отростки. Клеточное сообщество росткового слоя включает в себя однородные клеточные группы, находящиеся в цикле митотического деления (M), в стадии роста (B) и на этапе дифференцировки (D). Клетки эпидермиса выполняют различные функции и задачи. В нижней части эпидермиса, примыкая к дерме, располагается, так называемый, ростковый слой (зародышевый, базальный). Именно здесь происходит зарождение и становление новых клеток, обеспечивающих процессы обновления клеточных систем эпидермиса.

Часть зародившихся клеток, после подготовительного периода, опять включается в процесс размножения, а остальная часть клеток перемещается вверх и проходит этап роста (В), организуя основные клеточные органоиды и системы, необходимые для выполнения общих эпидермальных функций.

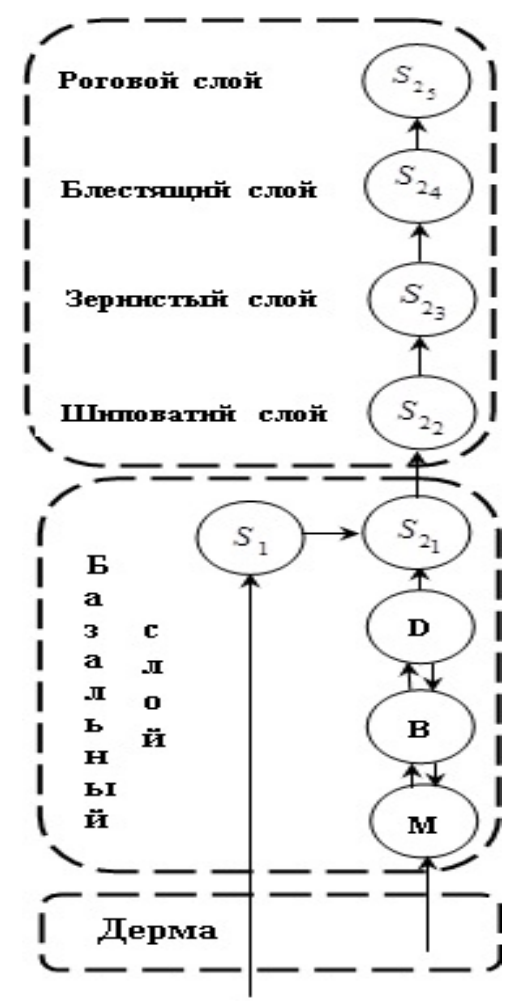

Рисунок 1 - Формализация структурно-функциональной организации эпидермиса (М - клетки митотического пула; В - растущие клетки; D - дифференцирующиеся клетки, $\mathrm{S}_{1}$ - меланиновые клетки; $\left\{\mathrm{S}_{2}\right\}$ - кератиновая группа клеток).

Эти клетки, в дальнейшем, дифференцируются (D) на кератиновые (S2 $\left.2_{1}\right)$ клеточные группы. Кератиновые клетки проходят ряд последовательных изменений в шиповатом $\left(\mathrm{S} 2_{2}\right)$, зернистом $\left(\mathrm{S} 2_{3}\right)$, блестящем $\left(\mathrm{S} 2_{4}\right)$, роговом (S25) слоях и вылущиваются во внешнюю среду. Надо отметить, что для сохранения дифференцировки меланоцитов обязательно присутствие кератиноцитов. Меланоциты являются важной составляющей клеточной популяции эпидермиса кожи человека, участвуют в сложных межклеточных взаимодействиях в тесной связи с кератиновыми клетками и под воздействием ультрафиолета образуют пигмент меланин. Кератиноциты и меланоциты относятся к иммунной системе кожи, продуцируя такие регуляторы как цитокины (информационные молекулы). Нарушения функционирования биорегуляторов иммунных реакций являются важными механизмами патогенеза псориаза, витилиго и рака кожи. Используя методику моделирования регуляторики клеточных сообществ функциональных единиц многоклеточных организмов и, приведя величины численности однородных групп клеток в текущий момент времени в соответствие с 
принятыми в данной статье обозначениями, мы можем написать следующие функциональнодифференциальные уравнения регуляторики численности клеточных сообществ эпидермиса:

$$
\begin{aligned}
& \frac{d M(t)}{d t}=\frac{a_{1} M^{m}(t-\tau)}{1+\prod_{i=1}^{5} S_{2_{i}}^{n}(t-\tau)}+b_{1} B(t-\tau)-a_{2} M(t) \\
& \frac{d B(t)}{d t}=a_{2} M(t-\tau)+b_{2} D(t-\tau)-\left(b_{1}+a_{3}\right) B(t) ; \\
& \frac{d D(t)}{d t}=a_{3} B(t-\tau)-\left(b_{2}+a_{4}\right) D(t) ; \\
& \frac{d S_{1}(t)}{d t}=a_{4} S_{2_{1}}(t-\tau)-a_{5} S_{1}(t) ; \\
& \frac{d S_{2_{1}}(t)}{d t}=a_{5} D(t-\tau)-a_{6} S_{2_{1}}(t) ; \\
& \frac{d S_{2_{i}}(t)}{d t}=a_{4+i} S_{2_{i-1}}(t-\tau)-a_{5+i} S_{2_{i}}(t) \\
& i=2,3,4,5
\end{aligned}
$$

где $M(t), B(t), D(t), S_{1}(t) u\left\{S_{2}(t)\right\}$ - величины, выражающие численности делящихся, растущих, дифференцирующихся, меланиновых и кератиновых однородных клеточных групп эпидермиса; $a_{1}-$ выражает скорость деления клеток в ростковом слое; $a_{5}$ - скорость гибели меланиновых клеток; $a_{8}-$ скорость гибели зернистых клеток; $a_{10}$ - скорость вылущивания клеток рогового слоя, остальные коэффициенты $\{a\},\{b\}$ выражают значения регуляторных параметров соответствующих клеток; $n$ степень сопряженности рассматриваемого клеточного сообщества эпидермиса. Значения всех коэффициентов - неотрицательны, что обеспечивает получение биологически разумных - неотрицательных решений системы приведенных уравнений. Следует отметить условность обозначения через $\mathrm{S} 24, \quad \mathrm{~S} 25$ численности клеток блестящего и рогового слоев. Предполагается, что в (1) посредством данных обозначений учитываются условные численности биологических образований (получающихся путем апоптоза клеток зернистого слоя) в блестящем и роговом слоях. Система уравнений (1) носит сложный характер и применение ее для качественного и количественного анализа механизмов функционирования клеток иммунной системы кожи требует разработки их модельных систем. Модельные системы, сохраняя основные свойства исходных уравнений, являются относительно простыми системами уравнений с минимально возможным числом соотношений и параметров. Это позволяет проводить, в большинстве случаев, успешный аналитический анализ характерных решений, а также определять основные режимы поведения рассматриваемых математических моделей. Рассмотрим модельную систему уравнений (1) применительно к анализу механизмов управления кератиновых клеточных сообществ эпидермиса. Предполагая предоставление ростковым слоем постоянного количества клеток шиповатогому слою за единицу времени ( $S_{2_{2}}=\mathrm{S}_{0}=$ const) систему (1) для зернистого, блестящего и рогового слоев можем написать в виде

$$
\begin{aligned}
& \frac{d S_{2_{3}}(t)}{d t}=a_{7} S_{0}-a_{8} S_{2_{3}}(t) ; \\
& \frac{d S_{2_{4}}(t)}{d t}=a_{8} S_{2_{3}}(t-\tau)-a_{9} S_{2_{4}}(t) ; \\
& \frac{d S_{2_{5}}(t)}{d t}=a_{9} S_{2_{4}}(t-\tau)-a_{10} S_{2_{5}}(t),
\end{aligned}
$$

с теми же обозначениями переменных и коэффициентов, что и в (1). Исследование системы (2) показывает, что, если повышается пролиферативная активность эпидермиса (увеличивается значение $S_{0}$, что например, наблюдается при псориазе, давление блестящего слоя к роговому слою тоже увеличится), то может привести к разрыву рогового слоя (происходит потеря содержимого блестящего слоя, раскрытие во внешнюю среду зернистого слоя, проникновение вредных веществ и микроорганизмов в эпидермис и т.д.). Определение конкретных характеристик решений (1), основных их режимов и свойств является трудоемким вследствие нелинейности рассматриваемых уравнений. Здесь актуально проведение качественного анализа функционально-дифференциальных уравнений типа (1), разработка и реализация методов построения приближенных решений (1) на РС.

Метод построения диаграмм Ламерея с вычислением энтропии Колмогорова, Хаусдорфового, информационного и более высших размерностей модельных систем уравнений (1) позволяет определять условия перехода к нерегулярности. Так, начинающийся процесс прогрессии опухоли кожи говорит о том, что трансформированная клетка миновала стадии торможения ее деления и гибели, а также «ускользнула» от системы противоопухолевого иммунного надзора. Дальнейшим последствием развития опухоли является синтез самой опухолью специфических факторов, подавляющих иммунный ответ на опухолевые агенты и лишающих иммунную систему возможности бороться с патологическим состоянием организма. $\mathrm{C}$ позиции 
нестабильности опухолевых клеток оценивается и качественно новое развитие опухоли, в частности активация или инактивация апоптоза и пролиферация (деление), в результате которых возникает биологическая прогрессия опухоли и приобретение ею новых клинических свойств, таких как агрессивность и высокая злокачественность. Область нерегулярных колебаний характеризуется нарушением системы регуляции организма с последовательным ухудшением функциональной активности. При этом могут наблюдаться случаи малых r-windows, внутри которых поведение клеток иммунной системы кожи носит нормальный характер. Размеры локальных неоднородностей r-windows в области динамического хаоса неодинаковы. Можно различать маленькие, средние и крупные регионы локальной неоднородности в области динамического хаоса рассматриваемых систем. Вход в r-windows в области динамического хаоса происходит резко, без всяких предшествующих признаков. В отличие от этого, выход из rwindows в область динамического хаоса происходит, как и первичное вхождение, после ряда бифуркаций (в которых показатель Ляпунова равен нулю) устойчивых колебаний. Следовательно, вход в область динамического хаоса (из основного и из малых регионов нормального поведения) легко заранее диагностируется, что может быть использовано в медико-биологической практике при диагностировании болезней, связанных с нарушением временных взаимоотношений в регуляционной цепи рассматриваемой системы организма. Количественные исследования показали, что основные характеристики rwindows (количество, размеры и местоположение) имеют нелинейный, сложный характер. Например, если параметры ресурсообеспечения и ассоциативности увеличиваются, это не обязательно означает, что число r-windows должно увеличиваться. Организм, используя адаптивные механизмы, может войти в ближайший регион регулярных решений. Это означает нормальную деятельность биосистемы. Дальнейшие аномалии в регуляции иммунной системы кожи могут привести к эффекту «черная дыра», к резким деструктивным изменениям в функционировании организма. Областями аномалий принято считать область динамического хаоса и область «черная дыра», Область динамического хаоса характеризуется нерегулярными колебаниями показателей функционирования динамических систем и может идентифицироваться как потеря регулирования в рассматриваемой системе. Она граничит с одной стороны с областью предельных циклов типа Пуанкаре (где поведение системы характеризуется двусторонне устойчивыми периодическими колебаниями), а с другой стороны - c областью резких деструктивных изменений - «черная дыра». Область деструктивных изменений может быть идентифицирована как начало необратимых кожных аномалий. Как показывают качественные и количественные исследования, поведение динамической системы в области «черная дыра» состоит из двух этапов: сильных нерегулярных колебаний и спад к тривиальному аттрактору. На втором этапе активность системы резко падает, и ее состояние можно определить по близости к тривиальному аттрактору. Численность клеток, после нескольких резких подъемов, необратимо падает. Этот режим соответствует конечной стадии заболевания.

\section{Conclusion}

Таким образом, разработанные математическое и программное обеспечения для анализа функционирования клеточного сообщества иммунной системы кожи при заболеваниях, а также методы качественного и количественного анализа построенных уравнений позволяют получать приближенные решения нелинейных дифференциальных уравнений с запаздыванием, оценивать поведение нерегулярных решений и «степень хаоса», количественно исследовать механизмы управления регуляторикой клеточных сообществ эпидермиса кожи в норме и при аномалиях. Особенно важны результаты анализа структурных особенностей области динамического хаоса, идентифицирование области с регулярным поведением r-windows в среде нерегулярных колебаний, поскольку наличие малых областей с нормальным поведением в области аномалий позволяет выбирать пути для вывода системы из области нерегулярного функционирования в область со здоровым состоянием.

\section{References:}

1. Voropaeva O.F., Shokin Y.I. (2012) Numerical simulation in medicine: Formulations of the

ISPC Innovations in science, 


\begin{tabular}{|c|c|c|c|c|c|c|}
\hline Impact Factor: & $\begin{array}{l}\text { ISRA (India) } \\
\text { ISI (Dubai, UAE } \\
\text { GIF (Australia) } \\
\text { JIF }\end{array}$ & $\begin{array}{l}=1.344 \\
=0.829 \\
=0.564 \\
=1.500\end{array}$ & $\begin{array}{l}\text { SIS (USA) } \\
\text { PИНЦ (Russia) } \\
\text { ESJI (KZ) } \\
\text { SJIF (Morocco) }\end{array}$ & $\begin{array}{l}=0.912 \\
=0.207 \\
=\mathbf{3 . 8 6 0} \\
=\mathbf{2 . 0 3 1}\end{array}$ & $\begin{array}{l}\text { ICV (Poland) } \\
\text { PIF (India) } \\
\text { IBI (India) }\end{array}$ & $\begin{array}{l}=6.630 \\
=1.940 \\
=4.260\end{array}$ \\
\hline
\end{tabular}

problems and some results of calculations // Computational technologies. 2012. V. 17. № 4. P. 29-55 (In Russ.).

2. Zhang H., Hou W., Henrot L., Schnebert S., Dumas M., Heusele C., Yang J. (2015) Modelling epidermis homoeostasis and psoriasis pathogenesis. // J R Soc Interface. 2015 Feb 6;12(103).

3. Pappalardo F, Forero IM, Pennisi M, Palazon A, Melero I, Motta S. (2017) SimB16: modeling induced immune system response against

B16-melanoma. PLoS ONE. 6(10) Article ID e26523.

4. Pennisi M. () A Mathematical Model of Immune-System-Melanoma Competition. Computational and Mathematical Methods in Medicine. 2012;2012:850754. doi:10.1155/2012/850754.

5. Adra S., Sun T., MacNeil S., Holcombe M., Smallwood R. (2010) Development of a three dimensional multiscale computational model of the human epidermis. // PLoS ONE. 2010, 5: e8511- 10.1371.
6. Laptev M.V., Nikulin N.K. (2010) A mathematical model of paracrine regulation of the proliferative activity of epidermis with the participation of T lymphocytes // Biophysics. April, 2010, Volume 55, Issue 2. P. 305-316.

7. Sheller B, D'Alessandro D. (2015) Analysis of a cancer dormancy model and control of immuno-therapy. Math Biosci Eng. 2015 Oct 1;12(5):1037-53.

8. Hidirov B.N. (2014) Selected works on mathematical modeling living systems regulatorika. Moscow-Ijevsk, 2014. 304 P. (in Russian).

9. Hidirov B.N. (2003) Modelling of Regulation Mechanisms of Living System // Scientiae Mathematicae Japonicae, 2003. Vol. 58. No 1. P. 419-425.

10. Hidirov B.N. (2008) Mathematical and Computer Modelling Regulatorika of Hierarchical Molecular-Genetic Systems // Scientiae Mathematicae Japonicae, 2008. Vol. 67. No 2. P. 229-239. 